\title{
Current Practice in Forefoot Surgery Among Foot and Ankle Surgeons in Spain
}

\author{
Mario Herrera-Perez ${ }^{\mathrm{a}, \mathrm{b}}$, Angeles Ayala-Rodrigo ${ }^{\mathrm{a}}$, Cesar Andarcia-Banuelos ${ }^{\mathrm{a}}$
}

\begin{abstract}
Background: Nowadays, there's an increasing interest in developing different minimal invasive (MIS) techniques in foot and ankle surgery although there's still little scientific supporting evidence. However, classic techniques in forefoot surgery, such as Keller procedure, are still used in selected cases.
\end{abstract}

Methods: We decided to perform a National Survey to help identify current practice in forefoot surgery between Foot Surgeons all over the country. We sent two questions via e-mail asking about surgeons' indications for MIS techniques and Keller procedure.

Results and Conclusions: The Keller procedure is still used in our country but in selected cases. MIS techniques are broadly used and most respondents chose the so called hybrid technique to solve hallux valgus deformity and metatarsalgia.

Keywords: Hallux valgus; Metatarsalgia; MIS techniques; Keller procedure

\section{Introduction}

Hallux valgus deformity and metatarsalgia are the most frequent pathologies seen in a Foot and Ankle Unit. Nowadays we have several reproducible surgical procedures, with good clinical evidence, to treat both problems after conservative treatment has failed [1].

With the development of Foot Surgery in the past twenty

\footnotetext{
Manuscript accepted for publication October 4, 2012

${ }^{a}$ Orthopaedic Department, University Hospital of Canary Isles, University of La Laguna, Tenerife, Spain

${ }^{\mathrm{b}}$ Corresponding author: Mario Herrera-Perez, Department of Orthopaedic Surgery. University Hospital of Canary Islands. Ofra, s/n.

La Cuesta, 38320. La Laguna, Tenerife (Canary Islands), Spain.

Email: herrera42@gmail.com
}

doi: http://dx.doi.org/10.4021/jcs113w years, there's an increasing interest in implementing minimally invasive surgery (MIS) techniques, that permit minimum soft tissue trauma and an earlier recovery of the patients. Though data is lacking to allow definitive conclusions on the use of these techniques for routine management of patients with hallux valgus $[2,3]$, it is quite worrying that these techniques are increasingly promoted with little if any critical supporting evidence [4].

On the other hand, some classic techniques, such as Keller procedure or excisional arthroplasty of the first metatarsophalangeal joint (resection of about a third of the proximal phalanx) are still used, especially in Europe, in spite of the high rate of complications described in the literature [5]. The Keller procedure was probably the most widely ever used bunion procedure, but due to the high incidence of incomplete correction and the associated postoperative transfer metatarsalgia, nowadays, excisional arthroplasty is recommended only for elderly sedentary patients with osteoarthritis of the first MTP joint in the absence of metatarsalgia preoperatively [2]. The purpose of this study was to assess the current state of practice among foot and ankle surgeons for operative treatment of common forefoot problems.

\section{Material and Methods}

One hundred and twenty Foot Surgeons were contacted via e-mail and asked to fill out an on-line questionnaire. Seventy two surgeons were members of the Spanish Society of Foot and Ankle Surgery, and the other forty eight were orthopaedic surgeons with special dedication to foot pathologies. The questionnaire was sent out in the period February to March 2011. All analysis was performed using Microsoft Excel 2007. The questionnaire is shown in Figure 1, we were also interested in finding out what the surgeons had to say in the additional comments.

\section{Results}

One hundred and twenty surgeons were contacted and we received a total of 92 replies (a response rate of 76.6\%). There 
1. In which situations do you use the Keller procedure in your clinical practice?

a. Never.

b. Only in cases of failed prior first ray surgery, as a salvage procedure.

c. Frequently in my practice.

d. Usually in hallux valgus or rigidus with the elderly.

e. Only in selected cases of degenerative hallux valgus or advanced hallux rigidus with the elderly and sedentary population.

COMMENTS:

2. In which situations do you perform the MIS technique (or percutaneous technique) in forefoot surgery in your clinical practice?

a. Never.

b. It's my "gold standard".

c. Only in mild deformities of the first ray.

d. Only to treat metatarsalgia of the second to fifth rays.

e. I use an "hybrid approach" : open surgery for the first ray and percutaneous for the lateral rays.

COMMENTS:

Figure 1. Questionnaire.

were two invalid responses, so the study group was defined as 90 .

\section{Past procedures: Keller technique}

A total of $44 \%$ of the respondents stated they never use Keller procedure in their practice (answer a); $40 \%$ perform this technique only in selected cases (answer e: degenerative $\mathrm{HV}$, advanced hallux rigidus, elderly and sedentary patients), $16 \%$ of the surgeons use the Keller procedure as a salvage technique in failed prior first ray surgery. We had only 5 additional comments: 2 surgeons preferred a metatarsophalangeal fusion instead of the Keller procedure and the other 3 surgeons try to preserve the joint using osteotomies of the first ray in spite of osteoarthritis of the first MTP joint.

\section{Present procedures: MIS Forefoot surgery}

A total of $33 \%$ of the surgeons never use the MIS and prefer the open procedures (answer a).

A total of $32 \%$ of the answers were $d$, they used the MIS technique for persistent metatarsalgia.

A total of $24 \%$ of the surgeons preferred an "hybrid procedure": open for the first ray and MIS for the lateral rays.
A total of $5 \%$ of the foot surgeons use the MIS technique in forefoot surgery as the "gold standard".

Only $6 \%$ of the surgeons use this technique in cases of mild HV deformity.

Therefore, $57 \%$ of the surgeons use some kind of percutaneous techniques in their clinical practice, meanwhile a $33 \%$ don't use this technique.

\section{Discussion}

Foot and ankle surgery has evolved in the past 20 years due to the development of modern implants and the understanding of anatomical and biomechanical concepts within this complex field. Hallux valgus is still one of the most common foot and ankle deformities treated by orthopaedic surgeons and correction of this deformity is one of the most common elective procedures undertaken on the foot [6].

Although it's very important for our field to improve the results of our surgeries with new methods of treatment, we cannot forget some of the "ancient" techniques that can be used in some instances. The Keller procedure represents the past in the Foot and Ankle Surgery, but, especially for young foot surgeons, should not be forgotten in our surgical arma- 
mentarium. Several Level IV retrospective case series have been published on the Keller resection arthroplasty [7-9], which demonstrated worse results with the Keller procedure than with proximal or distal first metatarsal osteotomies, with most of the patients with high rates of transfer metatarsalgia $[10,11]$. In spite of this, and according to Viladot et al, after a retrospective analysis of sixty-six feet operated on for hallux valgus, they conclude that the Keller procedure is still a simple and effective method for the surgical treatment of hallux valgus in elderly people with osteoarthritis at the first metatarsophalangeal joint [9]. Given that no more than Level IV evidence exists in the orthopaedic literature, only grade $\mathrm{C}$ evidence (conflicting or poor quality evidence) exists for recommending the Keller procedure in the management of hallux valgus deformity [2].

On the other hand, in the last few decades, several MIS techniques have been increasingly used and are available for minimally invasive correction of forefoot disorders. Theoretically, these techniques may provide better outcome for patients who would not recover well from traditional open approaches, because of decreasing recovery and rehabilitation times $[4,12]$, as surgical exposure and deep tissue dissection are smaller and gentler to the soft tissues. Nicola Maffulli et al performed a comprehensive search of articles published in peer reviewed journals and the results were clinically heterogeneous, so the authors conclude that "it's not possible to determine clear recommendations regarding the systematic use of MIS for hallux valgus correction, even though preliminary results are encouraging" [4].

According to the results of the survey, the Keller procedure is used in selected cases, especially with old and sedentary patients. Almost 2 out of 3 foot surgeons in our survey perform some kind of percutaneous surgery, mainly to treat metatarsalgia, with higher preference to traditional or open procedures for the first ray, and that seems likely to be the trend for the future, the use of the so called "hybrid techniques" in forefoot pathologies.

This study has limitations. First, many of the Foot Surgeons in our country may not have been surveyed. Second, we didn't receive too many comments to find out any other practice not included in the answers that we sent out.

\section{Conclusions}

The Keller procedure is still used in our country in selected cases and, in our opinion, should not be forgotten by the next generation of foot surgeons.

Only $5 \%$ of the foot surgeons of this survey use MIS techniques as their preferred technique, meanwhile one third of the surgeons never use MIS techniques.

MIS in foot and ankle is widely performed in our country: almost $60 \%$ of the foot surgeons in this study do some type of MIS or percutaneous surgery, with the "hybrid technique" being the preferred one in most of the cases.

\section{Conflict of Interest}

There are no conflicts of interest.

\section{References}

1. Fuhrmann RA, Roth A, Venbrocks RA. [Metatarsalgia. Differential diagnosis and therapeutic algorithm]. Orthopade. 2005;34(8):767-768, 769-772, 774-765.

2. Easley ME, Trnka HJ. Current concepts review: hallux valgus part II: operative treatment. Foot Ankle Int. 2007;28(6):748-758.

3. Louwerens JW, Valderrabano V, Winson I. Minimal invasive surgery (MIS) in foot and ankle surgery. Foot Ankle Surg. 2011;17(2):51.

4. Maffulli N, Longo UG, Marinozzi A, Denaro V. Hallux valgus: effectiveness and safety of minimally invasive surgery. A systematic review. Br Med Bull. 2011;97:149-167.

5. Coughlin MJ, Mann RA. Arthrodesis of the first metatarsophalangeal joint as salvage for the failed Keller procedure. J Bone Joint Surg Am. 1987;69(1):68-75.

6. Pinney S, Song K, Chou L. Surgical treatment of mild hallux valgus deformity: the state of practice among academic foot and ankle surgeons. Foot Ankle Int. 2006;27(11):970-973.

7. Majkowski RS, Galloway S. Excision arthroplasty for hallux valgus in the elderly: a comparison between the Keller and modified Mayo operations. Foot Ankle. 1992;13(6):317-320.

8. Turnbull T, Grange W. A comparison of Keller's arthroplasty and distal metatarsal osteotomy in the treatment of adult hallux valgus. J Bone Joint Surg Br. 1986;68(1):132-137.

9. Viladot R, Rochera R, Alvarez F, Pasarin A. [Resection arthroplasty in the treatment of hallux valgus]. Orthopade. 1996;25(4):324-331.

10. Schneider W, Knahr K. Keller procedure and chevron osteotomy in hallux valgus: five-year results of different surgical philosophies in comparable collectives. Foot Ankle Int. 2002;23(4):321-329.

11. Zembsch A, Trnka HJ, Ritschl P. Correction of hallux valgus. Metatarsal osteotomy versus excision arthroplasty. Clin Orthop Relat Res. 2000;(376):183-194.

12. Velazquez Pedroza VH, Lopez Marmolejo A, Isunza Ramirez A, Cortes Gomez J, Mora Rios FG, Mora Magana I. [Minimally invasive surgery efficacy in children's forefoot]. Acta Ortop Mex. 2008;22(1):19-25. 